\title{
Linear measurement and analysis of the gear rack
}

\author{
Biyun XIANG * \\ Shunde Polytechnic, Shunde Guangdong 528300,China \\ 492269219@qq.com
}

Keywords: linear measurement; laser interferometer ; motion controller;least square method; Fitting function.

\begin{abstract}
In the research of the linear motion of the gear drive mechanism, the measuring platform of the gear rack is designed in order to measure the linear error. using servo motor directly drives the gears on the rack. using servo motor directly drives the gear on the rack, and is based on the motion control PT point mode to realize the measurement of the Measuring distance and standby control requirements etc. In the process of the linear motion of the gear and rack drive mechanism, the measuring data is obtained by using the laser interferometer to measure the position of the actual movement of the gear axis. Using the least square method to solve the linear equations of contradiction, and to extend it to any number of times and arbitrary number of fitting functions, using MATLAB programming to obtain the actual data curve corresponds with design data curve, and the linear positioning accuracy and repeatability of gear and rack. This technology can be extended to linear measurement and data analysis of the majority of linear motion mechanism. It can also be used as the basis for the automatic compensation algorithm of linear motion control.
\end{abstract}

\section{Introduction}

In the field of industry, the application of gear rack drive mechanism is very wide. In mechanical equipment which requires heavy load, high precision, high rigidity, high speed, long distance movement control, gear and rack drive mechanism is applied to fast and accurate positioning. gear and rack drive mechanism is one of the important parts of industrial application.

At present, in the process of measuring the product measuring process of the gear and rack of most manufacturers, especially small processing factory, The common measurement method is the measurement of personnel survey instrument meter readings, or do some molding measure gauge. This method has limited accuracy, and it is also difficult to guarantee the accuracy of the gear rack. In order to improve the measuring method of gear rack, we try to innovate a kind of linear measurement and data analysis of the gear rack linear motion.

The design system for the production of straight teeth cylindrical gear rack meshing motion design a measurement platform, Control of servo motor drive gear on the rack with motion controller, A laser interferometer is used to measure the linearity of a gear at a selected set of nodes, Data processing after got data, The linear positioning accuracy and repeatability of the gear rack are obtained, Further research can be put forward to the control method of the gear rack movement parameters automatic compensation.

\section{Design measurement and analysis system}

The measurement system is composed of a measurement platform, a motion control system [1] and a laser interferometer [2] measuring system. As shown in Figure 1. On the aspect of data analysis of the gear rack, Matlab and its simulation software are used to analyze the ideal data of the gear rack [3], and on the other hand, the laser interferometer is used to measure and analyze the measured results. Finally, fitting the ideal and measured results, the adjustment method of the compensation of the rack and pinion motion parameters is presented. 


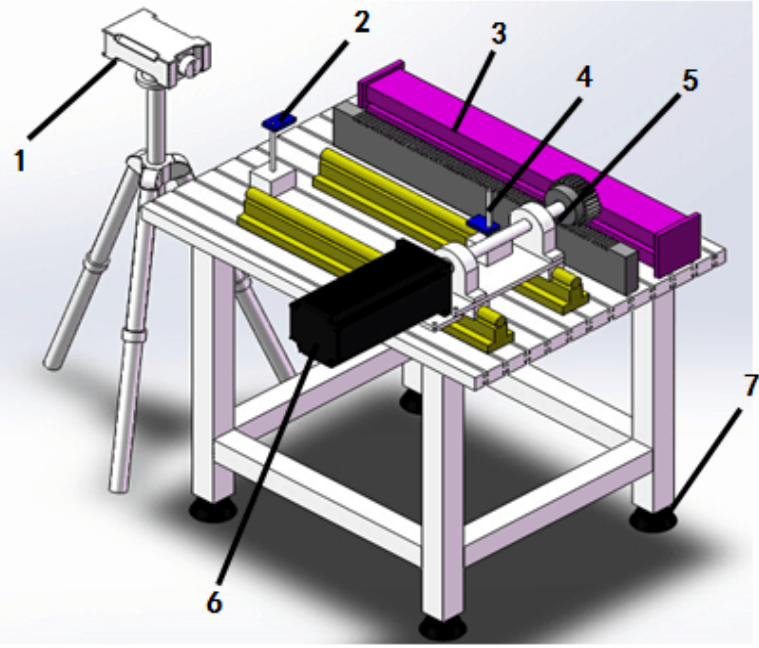

Figure 1 Measurement system

(1) Laser interferometer (2) Fixed mirror

(3) The moving mirror (4) Grating ruler

(5) Rack and pinion linear movement

(6) Motion control (7) Adjustable table

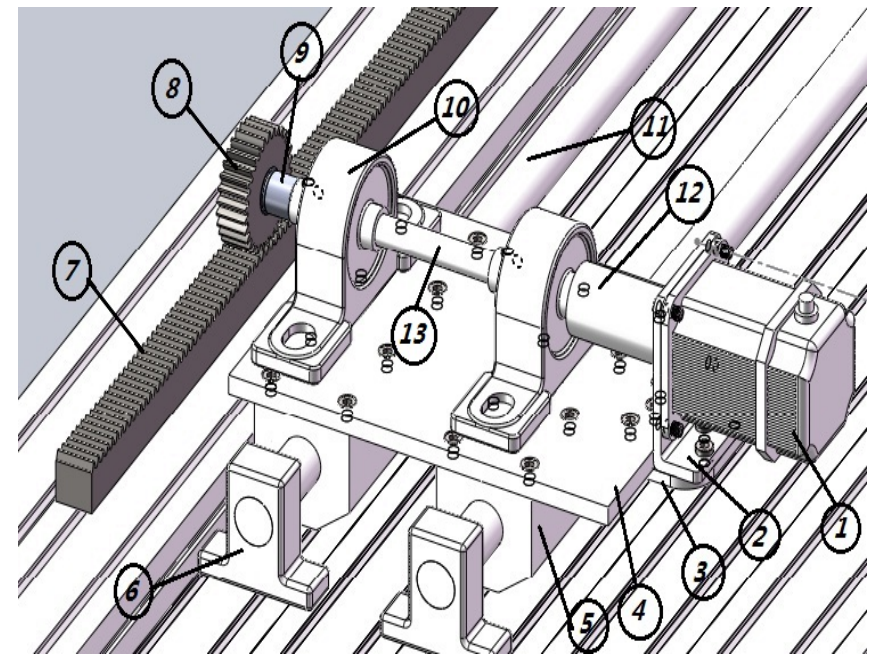

Figure 2 Mechanical structure

(1) Servo motor (2) Motor flange (3) The connecting angle

(4) The slide block (5) Slider (6) Motion mechanism

(7) Measured rack (8) Measured gear (9) Axle sleeve

(10) Ball bearing (11) Linear guide rail (12) Coupling

(13) Gear shaft

\section{Construction of measurement platform}

The measuring platform is composed of a gear rack and a mechanism for controlling the movement of gear and rack, The main mechanical structure [4] is shown in Figure 2.

\section{Collection of data of gear rack and rack}

This experiment selects the standard is 1.5 , the number of teeth is 30 standard spur gear, the modulus of the rack is also 1.5. In Figure 3, the node data of the rack and pinion is shown, Where the B, C, D, A point is Some nodes in meshing movement of gears and racks.

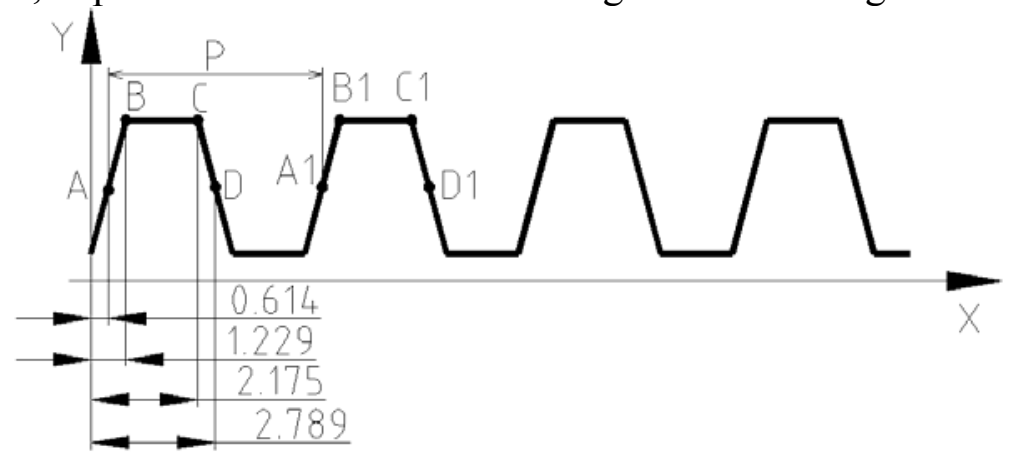

Figure 3 Gear rack and rack node data diagram

Design data of a gear rack:

Tooth distance: $\mathrm{p}=4.712 \mathrm{~mm}, \quad$ ( $\mathrm{a}$ is the distance between the measuring points), $(n=1,2,3 \ldots)(n<$ Number of teeth to be measured $/ \mathrm{a})$

$$
\begin{aligned}
& \mathrm{An}=\mathrm{A}+\mathrm{n} \cdot(a \cdot p) ; \\
& \mathrm{Bn}=\mathrm{B}+\mathrm{n} \cdot(a \cdot p) ; \\
& \mathrm{Cn}=\mathrm{C}+\mathrm{n} \cdot(a \cdot p) ; \\
& \mathrm{Dn}=\mathrm{D}+\mathrm{n} \cdot(a \cdot p) ;
\end{aligned}
$$

The motion controller controls the servo motor through the drive shaft to drive the gears on the rack, Using laser interferometer to do linear measurement[5], the actual data of gear movement is obtained. Gear movement to A point, pause, Measurement of A data with laser interferometer; 
then Control gear movement to A1 point, pause,Measurement of A1 data with laser interferometer; And then to the A2 point …... And so on, Until the complete bar is measured. The data for the An group. The numbers of $\mathrm{Bn}, \mathrm{Cn}$ and $\mathrm{Dn}$ groups were measured by this method. Select 4 node measurement data collected 4 sets of data.

\section{Data acquisition and motion control process}

The teeth are arranged on the platform as shown in Figure 1. The adjustment as far as possible in the rack and pinion gear indexing dot of the ideal, Then set up the laser interferometer, The distance between the selected teeth is measured as the distance, Linear measuring the meshing precision of gear and rack, The more accuracy of the gear and rack can be adjust by the analysis of the measured data.

In the experimental data measurement, we need to do the control requirements of the motion controller: Through the servo motor control gear movement to the first measurement point, and then stop; After 3S, the motor starts again, Control gear movement to second measuring points, and then stop, After $3 \mathrm{~S} \cdots \cdot \cdot$...And so on, Until the completion of all measurement points 。Control flow as shown in Figure 5.

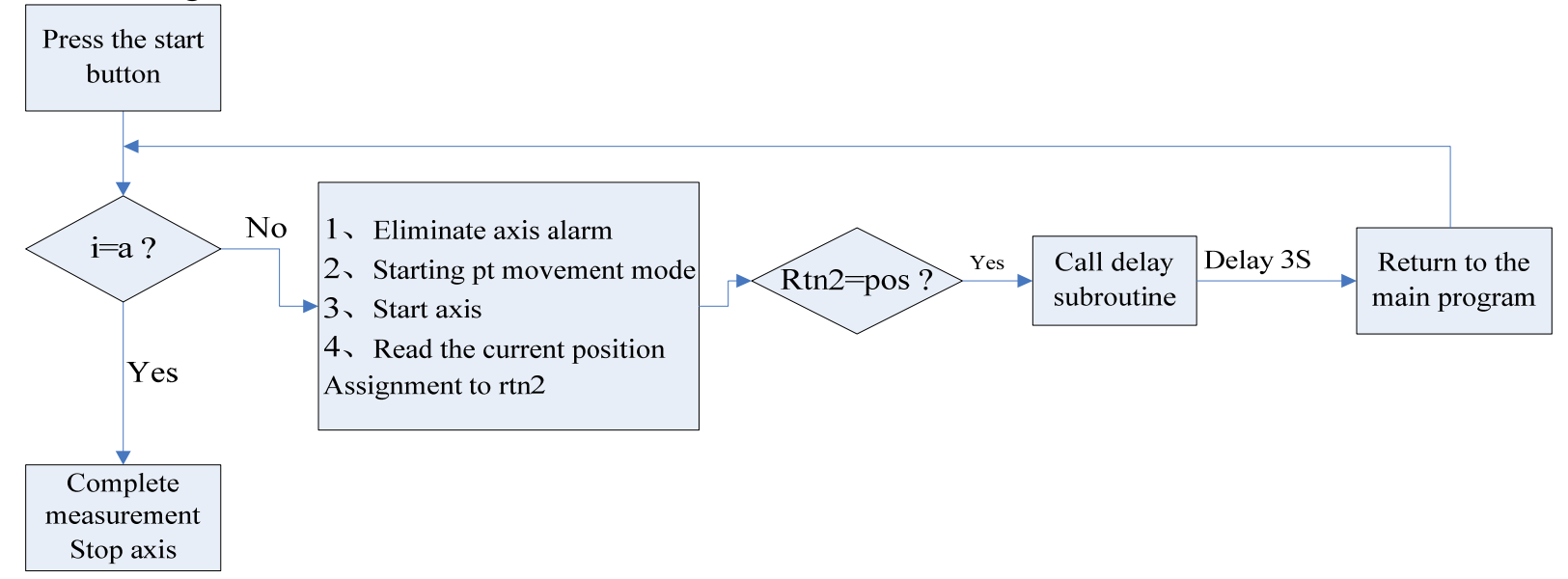

Figure 5 Motion control flow chart

\section{Establishment of data analysis model}

3.1 Fitting multivariate function with the solution of the contradiction equation

After the acquisition of the data is completed, the position error is analyzed according to the position curve of the data fitting. We obtain the fitting curve by solving the equation of the solution of the mean square error 。

From the mathematical derivation can prove: The solution of the equation $A^{T} \cdot A X=A^{T} Y$ is the solution of the contradiction equations $\mathrm{AX}=\mathrm{Y}$ in the sense of the least square method。

For $\mathrm{n}$ polynomial curve fitting, The minimum value problem for

$$
Q\left(a_{0}, a_{1}, \ldots, a_{n}\right)=\sum_{i=1}^{m}\left(a_{0}+a_{1} x_{i}+\ldots+a_{n} x_{i}^{n}-y_{i}\right)^{2},
$$

This is the solution to the contradiction equations:

$$
\left\{\begin{array}{l}
a_{0}+a_{1} x_{1}+a_{n} x_{1}^{2}+\ldots+a_{n} x_{1}^{n}=y_{1} \\
a_{0}+a_{1} x_{2}+a_{n} x_{2}^{2}+\ldots+a_{n} x_{2}^{n}=y_{2} \\
\cdots \\
a_{0}+a_{1} x_{m}+a_{n} x_{m}^{2}+\ldots+a_{n} x_{m}^{n}=y_{m}
\end{array} \quad \text { or } A \cdot\left[\begin{array}{l}
a_{0} \\
a_{1} \\
\ldots \\
a_{n}
\end{array}\right]=\left[\begin{array}{l}
y_{1} \\
y_{2} \\
\cdots \\
y_{m}
\end{array}\right]\right.
$$

The same solution to the minimal value problem of 


$$
\sum_{i=1}^{m}\left(a_{0}+a_{1} x_{i}+\ldots+a_{n} x_{i}^{n}-y_{i}\right)^{2} \quad A=\left[\begin{array}{cccc}
1 & x_{1} & \ldots & x_{1}^{n} \\
1 & x_{2} & \ldots & x_{2}^{n} \\
& \ldots & \ldots & \\
1 & x_{m} & \ldots & x_{m}^{n}
\end{array}\right] \text {, }
$$

So the $\mathrm{N}$ fitting curve of Discrete data $\left(x_{i}, y_{i}\right), i=1,2,3, \ldots, m$ is made $y=a_{0}+a_{1} x_{i}+\ldots+a_{n} x_{i}^{n}$, Can be obtained by solving the following equations:

$$
A^{T} A\left[\begin{array}{l}
a_{0} \\
a_{1} \\
\cdots \\
a_{n}
\end{array}\right]=A^{T}\left[\begin{array}{l}
y_{1} \\
y_{2} \\
\cdots \\
y_{n}
\end{array}\right]
$$

If the fitting function has $\mathrm{n}$ independent variables and a fitting, then the fitting function is:

$$
y=a_{0}+a_{1} x_{1}++a_{2} x_{2}+\ldots+a_{k} x_{k}+\ldots+a_{n-1} x_{n-1}+a_{n} x_{n}
$$

Pass $\mathrm{m}(\mathrm{m}>>\mathrm{n})$ time test, The experimental data of $\mathrm{M}$ group $\left(y_{i}, x_{1 i}, x_{2 i}, \ldots, x_{k i}, \ldots, x_{(n-1) i}, x_{n i}\right)$ were obtained, The equation of fitting function of the above $\mathrm{N}$ independent variables can be obtained (2) :

$$
\left(\begin{array}{ccccc}
m & \sum_{i=1}^{m} x_{i} & \sum_{i=1}^{m} x_{i}^{2} & \ldots & \sum_{i=1}^{m} x_{i}^{n} \\
\sum_{i=1}^{m} x_{i} & \sum_{i=1}^{m} x_{i}^{2} & \sum_{i=1}^{m} x_{i}^{3} & \ldots & \sum_{i=1}^{m} x_{i}^{n+1} \\
\ldots & \ldots & \cdots & \ldots & \ldots \\
\sum_{i=1}^{m} x_{i}^{n-1} & \sum_{i=1}^{m} x_{i}^{n} & \sum_{i=1}^{m} x_{i}^{n+1} & \ldots & \sum_{i=1}^{m} x_{i}^{2 n-1} \\
\sum_{i=1}^{m} x_{i}^{n} & \sum_{i=1}^{m} x_{i}^{n+1} & \sum_{i=1}^{m} x_{i}^{n+2} & \ldots & \sum_{i=1}^{m} x_{i}^{2 n}
\end{array}\right)\left(\begin{array}{l}
a_{0} \\
a_{1} \\
\ldots \\
a_{n-1} \\
a_{n}
\end{array}\right)=\left(\begin{array}{l}
\sum_{i=1}^{m} y_{i} \\
\sum_{i=1}^{m} x_{i} y_{i} \\
\ldots \\
\sum_{i=1}^{m} x_{i}^{n-1} y_{i} \\
\sum_{i=1}^{m} x_{i}^{n} y_{i}
\end{array}\right)
$$

As long as for equation (2) a little modification, We can obtain the equation of the fitting function of the random order of $\mathrm{n}$ independent variables(3), By solving the equation, we can get the coefficients of the fitting function.

$$
\left(\begin{array}{ccccc}
m & \sum_{i=1}^{m} x_{1 i} & \sum_{i=1}^{m} x_{2 i} & \ldots & \sum_{i=1}^{m} x_{n i} \\
\sum_{i=1}^{m} x_{1 i} & \sum_{i=1}^{m} x_{1 i} x_{1 i} & \sum_{i=1}^{m} x_{1 i} x_{2 i} & \ldots & \sum_{i=1}^{m} x_{1 i} x_{n i} \\
\ldots & \ldots & \ldots & \ldots & \ldots \\
\sum_{i=1}^{m} x_{(n-1) i} & \sum_{i=1}^{m} x_{(n-1) i} x_{1 i} & \ldots & \sum_{i=1}^{m} x_{(n-1) i} x_{(n-1) i} & \sum_{i=1}^{m} x_{(n-1) i} x_{n i} \\
\sum_{i=1}^{m} x_{i}^{n} & \sum_{i=1}^{m} x_{n i} x_{1 i} & \sum_{i=1}^{m} x_{n i} x_{2 i} & \ldots & \sum_{i=1}^{m} x_{n i}^{2 n}
\end{array}\right)=\left(\begin{array}{l}
\sum_{i=1}^{m} y_{i} \\
\sum_{i=1}^{m} x_{1 i} y_{i} \\
\ldots \\
a_{1} \\
a_{n-1} \\
a_{n}
\end{array}\right)\left(\begin{array}{l}
\sum_{i=1}^{m} x_{(n-1) i} y_{i} \\
\sum_{i=1}^{m} x_{n i} y_{i}
\end{array}\right)
$$

\section{Simulation validation of the model}

Fitting and Simulation of longitudinal data of gear:

Using the method of solving the contradiction equation, using a function to fit the Ideal coordinate data of the rack and pinion in the case $\mathrm{An}=\mathrm{A}+\mathrm{n} \bullet(a \bullet p)$; 且 $\mathrm{a}$ 取 10 , Take 7 times data acquisition, See Figure 3, As shown in Table 1, Recommended a fitting method. 
Table 1: Longitudinal data acquisition examples

\begin{tabular}{cccccccc}
\hline \multicolumn{7}{c}{ Longitudinal data measurement } \\
\hline $\mathrm{x}$ & 0.614 & 47.734 & 94.12 & 141.974 & 189.094 & 236.214 & 283.334 \\
$\mathrm{y}$ & 1.875 & 1.875 & 1.875 & 1.875 & 1.875 & 1.875 & 1.875 \\
\hline
\end{tabular}

Fitting function curve is $f_{(x)}=a_{0}+a_{1} x$ to form process equation:

$$
\begin{aligned}
& A^{T} A=\left(\begin{array}{cccc}
1 & 1 & \ldots & 1 \\
x_{1} & x_{2} & \ldots & x_{7}
\end{array}\right)\left(\begin{array}{cc}
1 & x_{1} \\
1 & x_{2} \\
\ldots & \ldots \\
1 & x_{7}
\end{array}\right)=\left(\begin{array}{cc}
7 & \sum_{i=1}^{7} x_{i} \\
\sum_{i=1}^{7} x_{i} & \sum_{i=1}^{7} x_{i}^{2}
\end{array}\right)=\left(\begin{array}{cc}
7 & 993.084 \\
993.084 & 203125.853
\end{array}\right) \\
& A^{T} Y==\left(\begin{array}{ccccccc}
1 & 1 & 1 & 1 & 1 & 1 & 1 \\
0.614 & 47.734 & 94.12 & 141.974 & 189.094 & 236.214 & 283.334
\end{array}\right)\left(\begin{array}{l}
1.875 \\
1.875 \\
1.875 \\
1.875 \\
1.875 \\
1.875 \\
1.875
\end{array}\right)=\left(\begin{array}{c}
13.125 \\
1862.0325
\end{array}\right)
\end{aligned}
$$

By the equation (1): $\quad\left(\begin{array}{cc}7 & 993.084 \\ 993.084 & 203125.853\end{array}\right)\left(\begin{array}{l}a_{0} \\ a_{1}\end{array}\right)=\left(\begin{array}{c}13.125 \\ 1862.0325\end{array}\right)$

Solution : $a_{0}=1.875, a_{1}=0, f_{(x)}=1.875$

Running and drawing in Matlab, Get the result of Figure 6, The left picture is the result of the operation, and the right is the fitting curve。
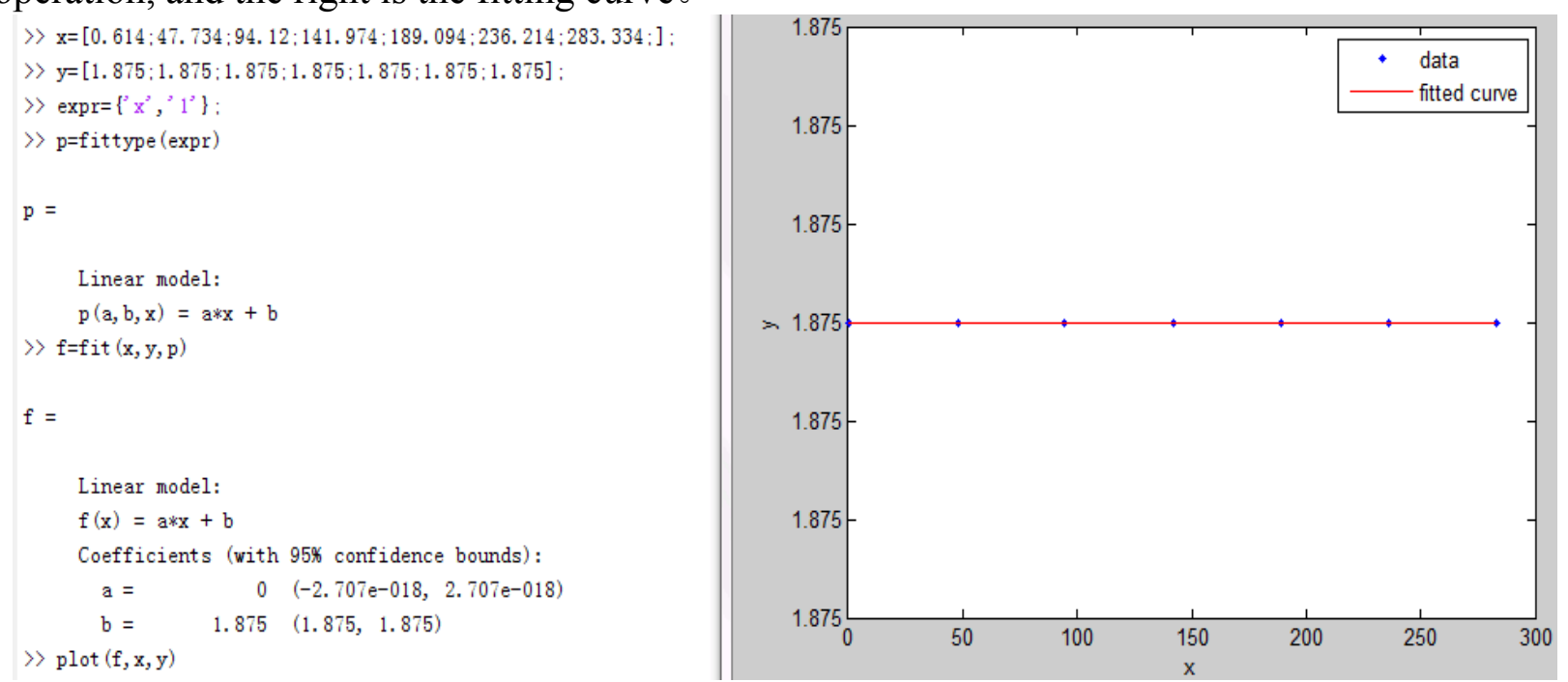

Figure 6 Longitudinal data running results and fitting curve

Fitting and Simulation of transverse data of gear:

Using the method of solving the contradiction equation, using a function to fit the Ideal data of the transverse coordinates of the gear and rack。The design of sliding distance is $10 \mathrm{~mm}$, Interval value of "a" is 10, Take 7 times data acquisition As shown in Table 2, Recommended a fitting method。 Table 2 Transverse data acquisition examples

\begin{tabular}{cccccccc}
\hline \multicolumn{7}{c}{ Transverse data measurement } \\
\hline $\mathrm{x}$ & 0 & 100 & 200 & 300 & 400 & 500 & 600 \\
$\mathrm{y}$ & 0.005 & 100.075 & 200.035 & 300.035 & 400.045 & 500.025 & 600.025 \\
\hline
\end{tabular}

Fitting function curve is $f_{(x)}=a_{0}+a_{1} x$ to form process equation, By the above equation method, the solution: $a_{0}=0.0023, a_{1}=0.9999, f_{(x)}=0.0023+0.9999 \mathrm{x}$ 
Running and drawing in Matlab, Get the result of Figure 7, The left picture is the result of the operation, and the right is the fitting curve.
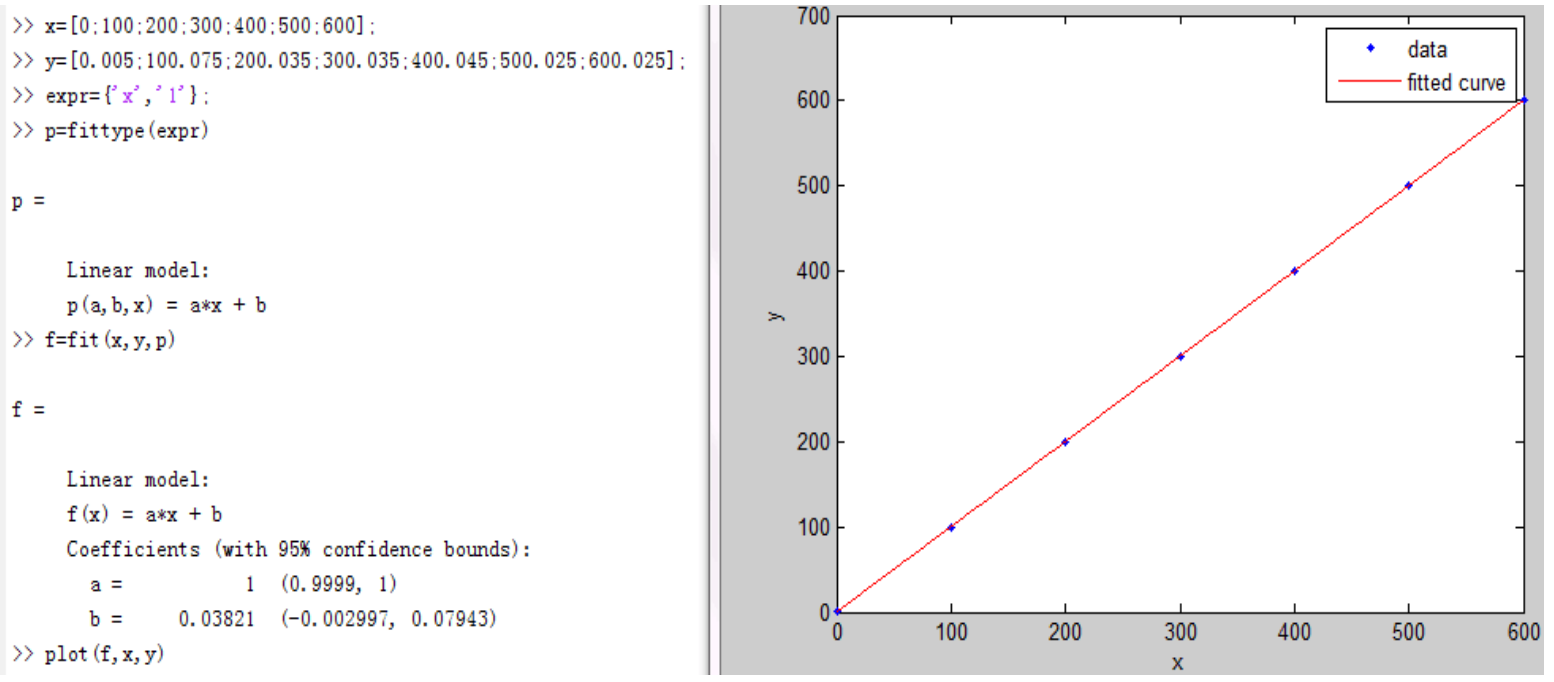

Figure 7 Transverse data running results and fitting curve

\section{Conclusion}

The design system for the production of straight teeth cylindrical gear rack meshing motion design a measurement platform, Control of servo motor drive gear on the rack with motion controller, A laser interferometer is used to measure the linearity of a gear at a selected set of nodes, Data processing after got data, The linear positioning accuracy and repeatability of the gear rack are obtained, Further research can be put forward to the control method of the gear rack movement parameters automatic compensation。In this set of equipment as long as we have a little modification of the measurement platform on the fixture and clamping method, it can be extended to the majority of linear motion mechanism to carry out linear measurement and data analysis, Have great application and popularization。The system used the laser interferometer measuring technology, If there is no laser interferometer, or without the need to measure the accuracy is very high, The position data of the grating ruler is read by the controller, and the measurement of the laser interferometer is replaced, it is a part of the project practicality to be improved。

\section{Acknowledgement}

In this paper, the research was sponsored by the 2013Foshan city science and technology innovation special funds Province (Project No. 2013AG100063).

\section{References}

[1] WANG Xianwei;CHENG Guangliang. CNC deep hole processing system based on motion controller applied research, .J. Journal of Changchun University1009-3907( 2015) 08-0001-03

[2] Xian Yimin. Applications of Laser interferometer,.J. Tool Engineering 1000-7008 (2015) 02-79-06

[3] Zhou Hao. The error analysis of linear data fitting method and its improved application, .J. College Mathematics 1672-1454 (2013) 01-0070-07

[4] Cheng Daxian The mechanical design manual, .S. 2008-05-01

[5] Renishaw. The principle and application of laser interferometer .M. 2004-02-01 